\title{
Elective bilateral above the knee amputation in T4-complete spinal cord injury: a case report
}

\author{
MS Jaffe \\ Department of Physical Medicine and Rehabilitation, Kaiser Permanente, San Diego, CA, USA
}

\begin{abstract}
Study design: This case study outlines an individual with a T4 complete level injury with Ashworth scale grade 4 spasticity of the lower extremities.

Objective: To discuss a treatment option for this individual with spinal cord injury ( $\mathrm{SCl}$ ) who had a variety of functional impairments, disabilities, and medical complications based on his level of injury. Setting: The department of physical medicine and rehabilitation at Kaiser Permanente in San Diego, California.

Methods: The patient's lower extremity spasticity contributed to pressure ulcers of his hips and feet as well as comfort and functional issues with his instrumental activities of daily living (ADLs). After failing medical therapies to control spasticity, and much discussion, bilateral above the knee amputations were performed.

Results: The patient had resolution of his $\mathrm{SCl}$ medical complications and a marked improvement in his quality of life.

Conclusion: This case study discusses a treatment option for a SCl-complete patient whose lower extremities had become detrimental to his function and general well being.

Spinal Cord (2008) 46, 585-587; doi:10.1038/sc.2008.58; published online 3 June 2008
\end{abstract}

Keywords: spinal cord injuries; amputation; elective surgical procedure

Spinal cord injury (SCI) affects approximately 250000 individuals in the United States. ${ }^{1}$ These individuals have a variety of functional impairments and disabilities based on their level of injury, the medical complications associated with their SCI and the specifics of each individual case. Such functional disabilities and SCI-associated medical conditions may have a profound effect on an SCI individual's perception of his/her quality of life (QOL). Some studies have shown that individuals with SCI when compared with a nondisabled community sample have a lower QOL. ${ }^{2,3}$

Spasticity is a common medical complication of SCI. The incidence is approximately 70\%, with roughly one-half of the patients requiring pharmacologic intervention. Spasticity may often lead to various complications including contractures, pain, impaired function and decreased QOL. ${ }^{4}$ In one study, $40 \%$ of SCI individuals with spasticity reported that their spasticity was problematic either by restricting activities of daily living (ADLs) or by causing pain, or both. ${ }^{5}$

Along with the effects of spasticity on ADL and pain, spasticity creates shear forces of the SCI person's limbs,

Correspondence: Dr MS Jaffe, Department of Physical Medicine and Rehabilitation, Kaiser Permanente, 4510 Viewridge Avenue, San Diego, CA 92123, USA.

E-mail: Michael.s.jaffe@kp.org

Received 25 March 2008; accepted 20 April 2008; published online 3 June 2008 which can lead to the development of pressure ulcers. These shear forces are magnified with functional impairments such as the sliding and dragging of limbs rather than lifting during transfers. ${ }^{4}$

The development of pressure ulcers is one of the most common and potentially serious complications of SCI. Among adults with SCI, the incidence of such ulcers ranges from 23 to $33 \%$ or more per year and up to $95 \%$ over the course of a lifetime. ${ }^{6,7}$ These SCI-associated pressure ulcers are one of the leading causes for hospitalization of individuals with SCI. The threat of pressure ulcers represents a potential challenge for the ability of patients with SCI to experience a full and satisfying life. ${ }^{8}$

This case study presents an individual with a T4-complete level injury who was 18 years out from his SCI. He was experiencing progressively adverse effects on his QOL due to difficulty in managing the spasticity and pressure ulcers associated with his SCI. We present a treatment option not previously discussed in the SCI literature involving elective amputation of the lower extremities.

\section{Case description}

A 49-year-old patient presented to our department of physical medicine and rehabilitation for out-patient 
consultation on 14 May 2004. The patient suffered a T4-complete SCI from a motorcycle accident in 1986. The patient lives at a modified independent lifestyle for all instrumental ADLs. The patient works as an insurance salesman and drives. The patient lives an active lifestyle, competing in adaptive triathlons and marathons.

At the start of 2002, the patient developed bilateral hip greater trochanter pressure ulcers and was followed closely by the plastic surgery department. He had multiple debridements and vacuum-assisted wound closure devices, and consideration was being made for a surgical flap procedure. The patient also suffered from a more recent right heel ulcer that developed 8 weeks before his visit to our department.

The patient had been referred to the department of physical medicine to discuss spasticity management. The patient states that while in the wheelchair, he had relatively few spasticity complaints. At night, however, he had severe spasticity, which fluctuated from extensor patterns to flexion patterns with his knees up to his chest. The shear forces of his spasticity seemed to be aggravating his pressure ulcer condition. The patient had been taking Baclofen $20-30 \mathrm{mg}$ at night to help control spasticity with minimal benefit. He had noticed some loss of bladder management with interruption of his continuous intermittent catheterization pattern due to some muscle relaxation associated with Baclofen use. The patient had noticed a dramatic increase in spasticity over the past year despite intermittent healing of his hip wounds.

On physical examination, the patient was a T4-paraplegia ASIA-A. He was a physically and mentally fit individual. He had full range of motion of all his major joints. He had a vacuum-assisted closure device on his right heel. His spasticity was rated at Ashworth scale 3-plus of the right lower extremity and four of the left lower extremity.

The initial treatment plan was to change the patient's oral Baclofen to Tizanidine at $12-24 \mathrm{mg}$ nightly. The patient was given reading material and a video about using an intrathecal Baclofen pump. An MRI of the patient's T-spine was ordered to rule out the possibility of post-traumatic syringomyelia. The patient was given a prescription for pressure relief, ankle foot orthotics to relieve heel pressure and to hopefully influence the extensor synergy pattern. A follow-up appointment was made for 6 weeks.

Upon the return visit, there was no change in the patient's condition. There was no change in his spasticity level and the MRI of the thoracic spine demonstrated only old SCI changes at the T4 level. The patient did not desire a trial of intrathecal Baclofen. It was during this visit that the patient brought up the possibility of undergoing bilateral above the knee amputations to improve his QOL. The patient felt that his lower extremities where heavy, difficult to control for self-care, affected his transfers and the spasticity of his legs exacerbated his functional impairments. He was also greatly concerned about the ongoing medical issues concerning his pressure ulcers.

A literature search was performed on PubMed with no case studies identified on the benefit of elective amputation in the SCI population. Over the course of 8 weeks, the patient and I had long discussions regarding the risks, benefits and alternatives to the surgery. We discussed the shift in his center of gravity, wheelchair seating issues and so on. The patient proved to be very considerate and reasonable. He obviously had given a good deal of thought on the topic and felt that bilateral above knee amputations would help his overall situation and function. We decided to refer the patient to an orthopedic surgeon.

The patient underwent bilateral above knee amputations on 4 August 2004. His postoperative convalescence was uneventful. The patient did not desire lower extremity prosthetics. He did receive a new manual ultralight wheelchair with a pressure relief cushion and composite wheels. All of the patient's spasticity issues and pressure sore issues resolved within 6 months of the amputation.

The patient returned to our clinic on 19 June 2007 to request a new cushion and breaks for his wheelchair. The patient commented on how happy he was with the outcome of the amputations stating that it was 'the best decision I had ever made'. The patient continued to be modified independent for all his instrumental ADLs. The patient was participating in long distance hand cycling trips of up to 700 miles. He has had no recurrence of lateral hip pressure ulcers and no issues with spasticity.

\section{Discussion}

The quality of life issues in the SCI population are a selfreported and subjective measure that is dynamic in nature and multidimensional. It may be difficult, as a physical medicine and rehabilitation physician to look at a clinical scenario through the patient's eyes when discussing their medical issues. In the case of SCI, our training as rehabilitation physicians biases us to strive for tissue preservation and maximizing a patient's abilities and functions given a patient's physical attributes and abilities. When presented with medical complications from an existing physical disability, our initial goal, as clinicians, is to treat that medical complication through existing accepted medical treatments. In this case, severe spasticity and pressure ulcers were present as a complication of long-standing SCI. This individual failed to respond to attempts at controlling his spasticity and prevent pressure ulcers from forming. The patient, in this case, realizing the limitations of traditional treatment modalities and practices for his condition came up with his own idea for a solution. Engaging in a healthy discussion with his care providers, a reasonable alternative treatment plan was devised. The patient wished to have bilateral above the knee amputations of his asensate and plegic lower extremities. Though this treatment plan did not initially sit well with his rehabilitation physician, time and lengthy discussions regarding expectations and potential risks won over the physiatrist's feelings of unease.

In this case study, the patient's SCI-associated spasticity and pressure ulcers were alleviated by the above the knee amputations. The patient's QOL was dramatically improved along with his functional abilities according to the patient.

This case study by no means suggests amputation as an aggressive treatment for the control of SCI-associated 
spasticity and pressure ulcers. However, this case does narrate a possible treatment option for selected SCI individuals.

More importantly, this case is meant to outline the working relationship regarding treatment options between a clinician and patient.

\section{Conclusion}

This case study outlines the potential benefit of elective amputation in the SCI-complete population. SCI medical complications and functional impairments may arise in the limbs below the level of the SCI. These medical issues and impairments may not be adequately addressed with traditional therapies. If medical therapy fails, and QOL issues dictate, the alternative of amputation of the limbs may be a prudent choice in selected cases.

\section{Acknowledgements}

I thank Linda Jaffe, MD, for draft review and Linda Masliah at our health science library.

\section{References}

1 National Spinal Cord Injury Statistical Center. Spinal Cord Injury Information network. University of Alabama at Birmingham's Department of Rehabilitation Medicine. 2007. http://main. uab.edu/show.asp?durki $=10766$

2 Westgren N, Levi R. Quality of life and traumatic spinal cord injury. Arch Phys Med Rehabil 1998; 79: 1433-1439.

3 Kreuter M, Sullivan M, Dahllof A, Siosteen A. Partner relationships, functioning, mood and global quality of life in persons with spinal cord injury and traumatic brain injury. Spinal Cord 1998; 36: 252-261.

4 DeLisa J, Gans B. Rehabilitation of Spinal Cord Injury. Physical Medicine and Rehabilitation Principles and Practice, 4th edn. Lippincott Williams and Wilkins: Philadelphia, PA, USA, 2005.

5 Camilla S. Spasticity after traumatic spinal cord injury: nature, severity, and location. Arch Phys Med Rehabil 1999; 80: $1548-1556$.

6 Garber SL, Rintala DH, Hart KA, Fuhrer MJ. Pressure ulcer risk in spinal cord injury: predictors of ulcer status over 3 years. Arch Phys Med Rehabil 2000; 81: 465-471.

7 Fuhrer MJ, Garber SL, Rintala DH, Clearman R, Hart KA. Pressure ulcers in community-resident persons with spinal cord injury: prevalence and risk factors. Arch Phys Med Rehabil 1993; 74: 1172-1177.

8 Clark FA, Jackson JM. Data-based models of how pressure ulcers develop in daily-living contexts of adults with spinal cord injury. Arch Phys Med Rehabil 2006; 87: 1516-1525. 